\title{
An Interactive Auto-recharging System for Mobile Robots
}

\author{
Kuo-Lan Su1, *, Yi-Lin Liao², Shih-Ping Lin ${ }^{3}$ and Sian-Fu Lin ${ }^{3}$ \\ ${ }^{1}$ Department of Electrical Engineering, National Yunlin University of Science \& Technology, Taiwan \\ ${ }^{2}$ Graduate School of Engineering Science and Technology, National Yunlin University of Science \& Technology, Taiwan \\ ${ }^{3}$ Graduate School of Electrical Engineering, National Yunlin University of Science \& Technology, Taiwan \\ (Received 2 March 2013; Accepted 3 May 2013; Published on line 1 March 2014) \\ *Corresponding author: sukl@yuntech.edu.tw \\ DOI: 10.5875 /ausmt.v4i1.197
}

\begin{abstract}
The article describes a new auto-charging system designed to enhance successful docking rates for mobile robots while at the same time monitoring the status of the charging current between the mobile robot and the docking station. The communication interface of the mobile robot and the docking station uses a wireless RF interface. The docking station is designed with one active degree of freedom and two passive degrees of freedom. Users tune the location of the charging pins on the docking station to make it possible for the mobile robot to execute an auto-recharging process. The auto-recharging process uses multiple sensors and a laser range finder located on the mobile robot. The laser range finder searches for a landmark to guide the mobile robot towards the docking station. In the experiment, the power of the mobile robot is under its threshold value. The mobile robot transmits a charging command to the docking station via a wireless RF interface. The docking station transmits a location command back to the mobile robot via this wireless RF interface. The mobile robot uses its laser range finder to search for the landmark of the assigned docking station and programs a motion trajectory to move forward to the docking station. The docking station supplies a charging current to the mobile robot by means of a charger. The power detection module simultaneously detects the charging current and the voltage values of the charging process in both the docking station and the mobile robot. The system can monitor the status of the charging process between the docking station and the mobile robot at any time. The power of the mobile robot is enough to be detected by the power detection module. When the charging process is complete, the docking station turns off the charging current and triggers the mobile robot to leave the docking station via the wireless RF interface.
\end{abstract}

Keywords: auto-charging system; mobile robots; docking station; laser range finder; wireless RF interface

\section{Introduction}

With the development of robotic technologies over the years, mobile robots have been widely applied in many fields such as factory automation, dangerous environment detection, office automation, hospital service, entertainment, space exploration, farm automation, and fishery automation, and in military, education and security systems, and so on. In general, researchers ignore the important issue of the residual working time of mobile robots and their power detection systems. When the power of a mobile robot falls under its threshold value it must execute an auto-recharging process in real-time; otherwise it cannot be controlled by commands, and some dangerous event may occur. This paper describes the development of an auto-recharging process for mobile robots. The mobile robot uses its power detection module to detect power sources at any time, computes its residual working time, and searches for the landmark of a docking station using a laser range finder. 
We designed a power detection module and applied it to mobile robots using a microprocessor (MCS51), and the on-line experimental results are very successful [1]. Recently we designed a new power detection module and applied it to a fire fighting robot using a HOLTEK microchip $[2,3]$. The goal of the module was to detect multiple power values simultaneously, to reduce the cost of the power detection module, to extend the interface function to transmit measurement information to the main controller of the mobile robot via a series interface, and to reduce error detection using redundant management methods and a statistical estimation method [4]. We extended the functions of the power detection module so that they could be applied to the auto-recharging monitoring system of the mobile robot. In the past many research studies have proposed different power detection methods. Horning and Sodden reported on numerous experiments where current measurements were used to forecast reliability problems in devices which had previously passed conventional test procedures $[5,6]$.

In recent years, many auto-recharging systems and power detection methods have been proposed. Liu

Kuo-Lan Su received B. S. and M. S. degrees in automatic control engineering from Feng-Chia University, Seatwen, Taiwan, R. O. C., and received a PH.D.degree in electrical engineering at National Chung-Cheng University, Chia-Yi, Taiwan, R. O. C., He is currently an associate professor in the Department of Electrical Engineering, National Yunlin University of Science \& Technology. His research interests include multi-sensor fusion algorithms, automation design, control algorithms and robotics.

Yi-Lin Liao received a M. S. degree in the department of electrical engineering, from National Yunlin University of Science \& Technology, Yunlin , Taiwan, R. O. C. He is currently a student in the Graduate school of Engineering Science and Technology at the National Yunlin University of Science \&Technology, Douliou, Yunlin, Taiwan, R. O. C. His research interests include multi-sensor fusion algorithms, applications of the HOLTEK microchip and robotics.

Shih-Ping Lin received his B.S. degree (2009) from the Department of Electrical Engineering at the National United University. In college, he served as the archives propaganda department head of the student association. His monograph was "Fault Diagnosis of Transformers." Since 2011 he has been enrolled at the National Yunlin University of Science and technology, and joined the master's course in the Department of Electrical Engineering, Yunlin, Taiwan. The main researching fields that he has been involved in include 8-bit MCU control, mobile robots and sensor applications. His research interests include electronic circuit design, $\mathrm{PCB}$ layout, 8-bit $\mathrm{MCU}$, sensor applications, and $A D C$ circuits. He has also acquired an AutoCAD international license. He is now studying "Motion Planning of Multi-Docking Systems for Intelligent Mobile Robots."

Sian-Fu Lin received his B.S. degree (2011) from the Department of Electrical Engineering at the National Yunlin University of Science \& Technology. He has excellent practical skills in the field of automatic control, and with interpersonal groups in social relationships. In 2011 while in college, he received the Certificate for Automation Engineer from the Taiwan Automation Intelligence and Robotics Association (TAIROA) in 2011, and also served as leader of the Artistic Flower Design Club. Since 2011 he has been enrolled in the National Yunlin University of Science and Technology master's course in the Department of Electrical Engineering. His main research activities have been in sensor applications and control in 8-bit Microcontrollers. His research interests include electronic circuit design, PCB layout, sensors, $A D C$ and compiling firmware. He is now studying the "Development of the Driver Systems of Auto-Detection Devices". developed a new sensor module for the UBot self-reconfigurable robot system and proposed a novel docking method for precise docking employing a module group with a sensor module and a target module [7]. Ren developed a docking control method which is based on vision feedback and is applied in a narrow space (a U-shaped bed) [8]. Shen proposed a vision-based robot which was used to deal with the battery swapping work of an electric bus [9]. Bai proposed the design of an autonomous robotic vacuum cleaner by using three-dimensional vector coordinates to guide its path when it moved to the docking station [10].

A continuing question is how to guide a mobile robot to move forward to its docking station. The structure of the docking station and the docking method are very important. In past literature, some experts have researched auto-recharging systems for mobile robots. Silverman designed a new docking station for the Pioneer 2DX mobile robot and presented a method to provide long-term autonomy by implementing autonomous recharging [11]. The first work on robot recharging was made by Grey Walters [12]. Raufas developed the first autonomous recharging mobile robots by employing a light to find a hut, which contained a light beacon and a battery recharger, and used four IR LED emitters and one IR receiver to implement the docking [13]. Arkin programmed a docking method to allow 6 degrees of error. The robot can approach the recharging station using a map and makes contact with a specially designed battery recharging system [14]. Some systems are using vision-based methods to implement docking $[15,16]$.

\section{Architecture}

The auto-recharging system described in this paper integrates a docking station and a mobile robot. The system architecture of the docking station has several parts. One is a power detection module. The others are a charger, a structure and a landmark, and the wiring shown in Figure 1 . The power detection module includes some sensory devices, some driver devices, and a wireless RF module. The sensory devices include input signals containing four current measurement and four voltage measurement signals. A limit switch detects whether the mobile robot has touched the docking station or not and transmits a digital signal to the controller of the power detection module. The driver device contains a relay device and an LCD display panel and controls the charging current output. The power detection module measures the status of the charging current, such as over loading (short), no current (shutoff) or normal current. The wireless RF module transmits and receives the charging process commands exchanged with 
the mobile robot. The landmark can guide the mobile robot to search for and locate the docking station. The charger supplies the charging current to the mobile robot through a relay device.

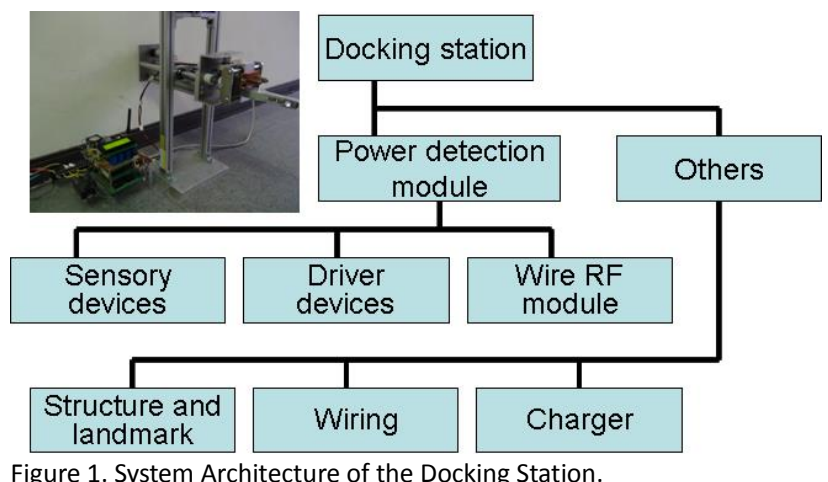

The mobile robot has the shape of a cylinder and its diameter, height, and weight are $40 \mathrm{~cm}, 60 \mathrm{~cm}$, and 30 $\mathrm{kg}$, respectively. It is equipped with an IPC (Industry Personal Computer) as the main controller. The parts of the mobile robot are shown in Figure 2. One part is the main controller. The other parts include a charging device, a structure, wiring and a power system. The main controller can program the motion trajectory of the mobile robot in relation to the docking station and contains a wireless RF module and a detection system. The module based detection system includes systems for obstacle detection, environment detection, and power detection as well as a laser range finder.

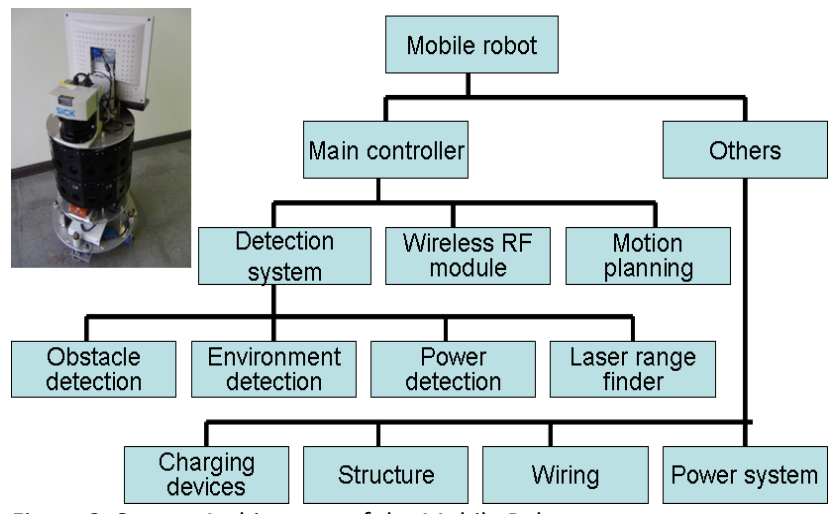

Figure 2. System Architecture of the Mobile Robot.

The laser range finder searches for the landmark of the docking station and guides the mobile robot's movement towards the docking station. The environment detection system contains GSM modern and other modules. These modules include a fire detection module, an intruder detection module and temperature and humidity detection modules. The obstacle detection system contains sixteen ultrasonic sensors and sixteen IR sensors. The wireless RF interface contains a wireless RF module and a wireless LAN. The wireless RF module communicates with the docking station and transmits the control commands of the charging process from the docking station. The wireless LAN communicates with a remote supervising computer. Meanwhile, the structure of the mobile robot has four wheels to provide the capability of autonomous mobility. An auto-switch controls the recharging current from the charger of the docking station. The power system consists of batteries which are used to supply various amounts of power through DC/DC conversion to all of the mobile robot's devices.

The power detection module monitors power variations in the charging process and measures the exact power values of the mobile robot, as shown in Figure 3. The controller of the power detection module is a HOLTEK microchip which is also the controller of the docking station. The power detection module detects the charging current in the mobile robot and the docking station using redundant management methods and statistical estimation methods. It also receives the signals from the limit switch and wireless RF module in the docking station, and communicates with the main controller of the mobile robot via a series interface (RS232). The main controller can predict residual working time using an auto-regressive algorithm derived from measurement information provided by the power detection module that is embedded in the mobile robot.

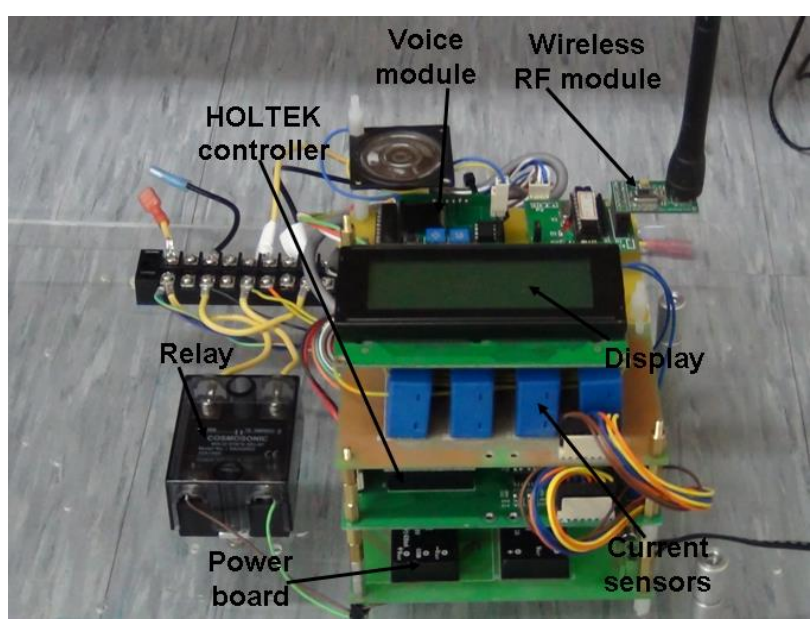

Figure 3. Power Detection Module.

\section{Docking station}

The docking station provides two contact points (charging pins) to connect with the charging connection structure of the mobile robot. The charging points are assigned to connect with the batteries of the mobile robot through an auto-switch device. A guiding stick is mounted on the docking station to guide the insertion of the charging pins into the charging holes. The docking station is designed with two passive DOFs (Degree Of Freedom) and one active DOF. In the two passive DOFs, it rotates in the $Z$-axis and moves in the $X$ axis, and uses a 
compression spring to tune on two various docking conditions for the mobile robot. The controller of the power detection module controls the charging pins moving in the $Y$ axis by means of the active DOF. Users can adjust the height of the charging pins so that they connect with the charging holes on the mobile robot. The charging connection structure of the mobile robot is mounted in the same direction as the laser range finder. The prototype of the docking station is shown in Figure 4.

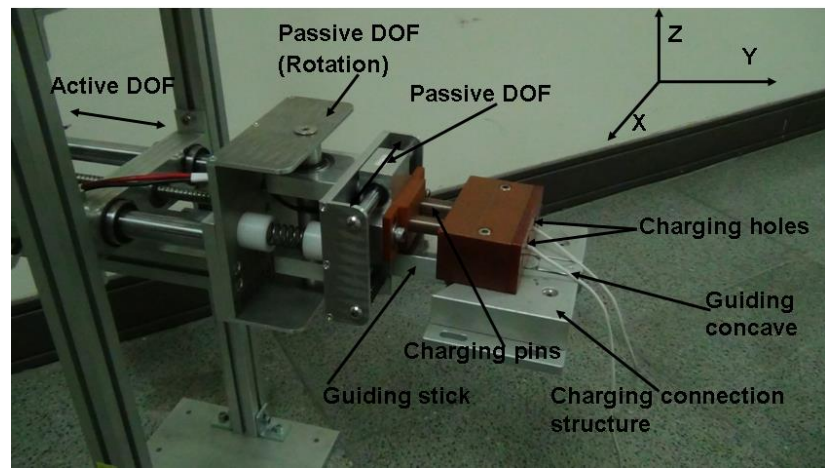

Figure 4. Prototype of the Docking Station.

The charging adapters of the mobile robot are on the inside of the charging holes. Each hole is shaped as a cone in order to help the docking take place smoothly. Since both the connection pins and adapters are rigid, the docking station is designed to comply with reasonable robot docking offset angles and offset displacements. When the mobile robot is approaching the docking station at an offset angle and with offset displacement, the guiding stick can adjust the offset error to guide the charging pins to connect with the charging adapters.

Figure 5(a) shows the mechanism of the docking station. Extension sprints are used to bring the charging pins back to the center after the robot disengages. A small entry offset and angle are allowed when the robot is in the docking process. The maximum offset rotational angle and horizontal offset are 5 degrees and $2 \mathrm{~cm}$ respectively. We show a sliding bar to overcome an error distance in the $X$ axis on the docking station in Figure 5(b). A pilot is embedded on the docking station to slide into the concave receptor in the mobile robot. After the robot leaves the docking station, compressed springs on the sliding bar are used to bring the pilot to center. $A$ rotational bar to overcome an angle in the $Z$ axis on the docking station is shown in Figure 5(c). The pilot on the docking station slides into the concave receptor in the mobile robot. After the robot leaves the docking station, compressed springs on the rotational bar are used to bring the pilot to center.

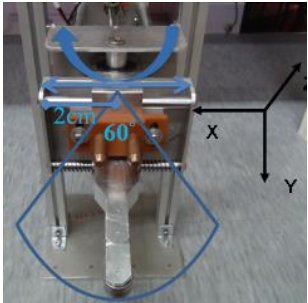

(a)

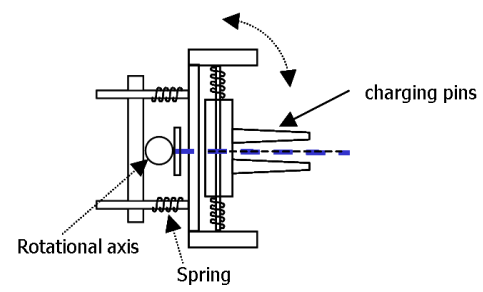

(b)

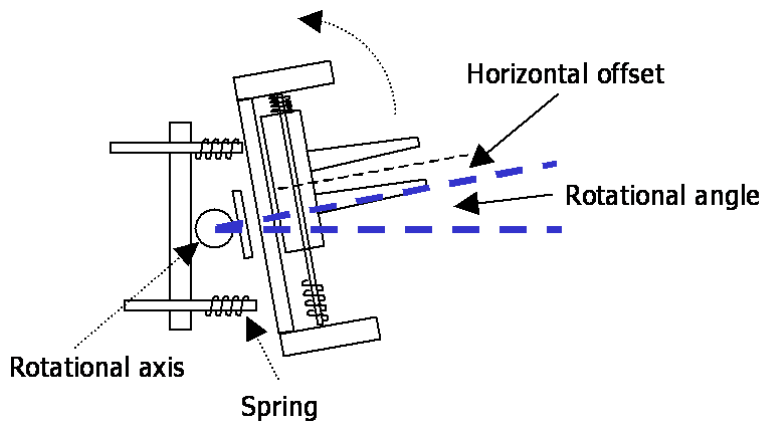

(c)

Figure 5. Docking Methods.

We show two examples of docking methods under real conditions. Figure 6(a) shows a small entry docking offset displacement on the left side for the mobile robot as it moves forward to the docking station. Extension sprints are used to bring the charging pins back to the center after the robot disengages as shown in Figure 6(b). Then the guiding stick slides into the guiding concave of the charging connection structure, and the charging pins move into the charging holes very smoothly as shown in Figure 6(c).

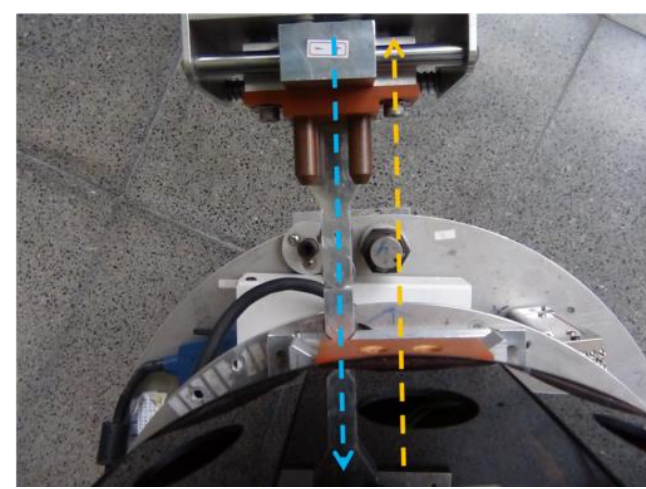

(a)

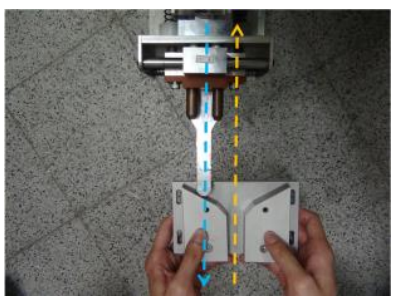

(b)

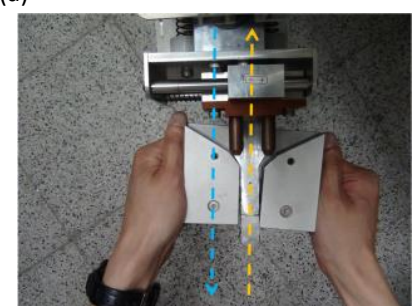

(c)
Figure 6. Docking Processing with Offset Displacement. 
Figure 7(a) shows a slight entry offset angle as the mobile robot moves forward to the docking station. The mobile robot executes the charging process. The guiding stick of the docking station touches the right side of the mobile robot and rotates on the $\mathrm{Z}$ axis to slide into the guiding concave, as shown in Figure $7(\mathrm{~b})$ and (c). In general, the mobile robot moves forward to touch the docking station. The offset angle and offset displacement may happen simultaneously. The guiding stick can guide the charging pins so that they insert into the charging holes of the mobile robot very smoothly.

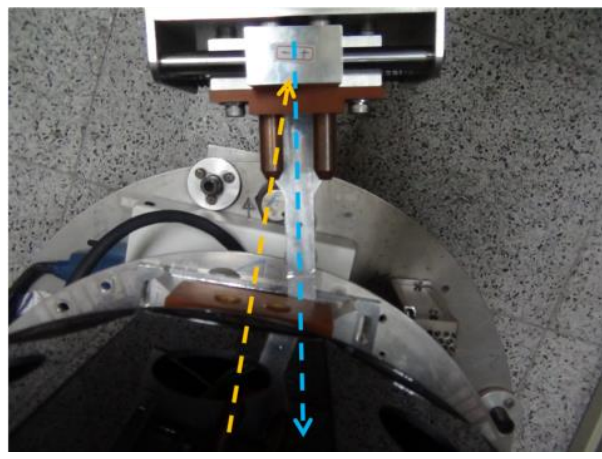

(a)

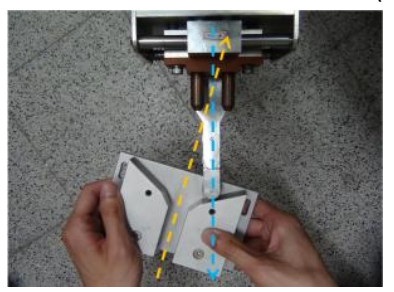

(b)

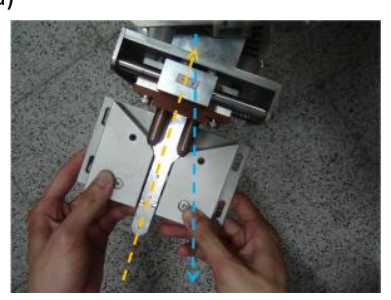

(c)
Figure 7. Docking Processing with Offset Angle.

The auto-recharging process is presented in two parts, as shown in Figure 8. One is described in relation to the docking station; the other is described in relation to the mobile robot. We list the relationship commands between the docking station and the mobile robot via wireless RF interface to execute the auto-recharging process. The power detection module of the mobile robot detects that power is under the threshold value for moving for a long time in free space. The mobile robot transmits an RF signal to the docking station and executes the charging process to search for the landmark of the assigned docking station. The docking station receives the wireless RF signal, and transmits the location to the mobile robot. The mobile robot moves to the docking station using its laser range finder.

The auto-recharging process is presented in two parts, as shown in Figure 8. One process relates to the docking station; the other relates to the mobile robot. We list the commands between the docking station and the mobile robot via wireless RF interface relating to the auto-recharging process. The power detection module of the mobile robot detects that power is under the

threshold value for moving for a long time in free space. The mobile robot transmits the RF signal to the docking station and executes the charging process to search for the landmark of the assigned docking station. The docking station receives the wireless RF signal and transmits the location to the mobile robot. The mobile robot moves to the docking station using the laser range finder.

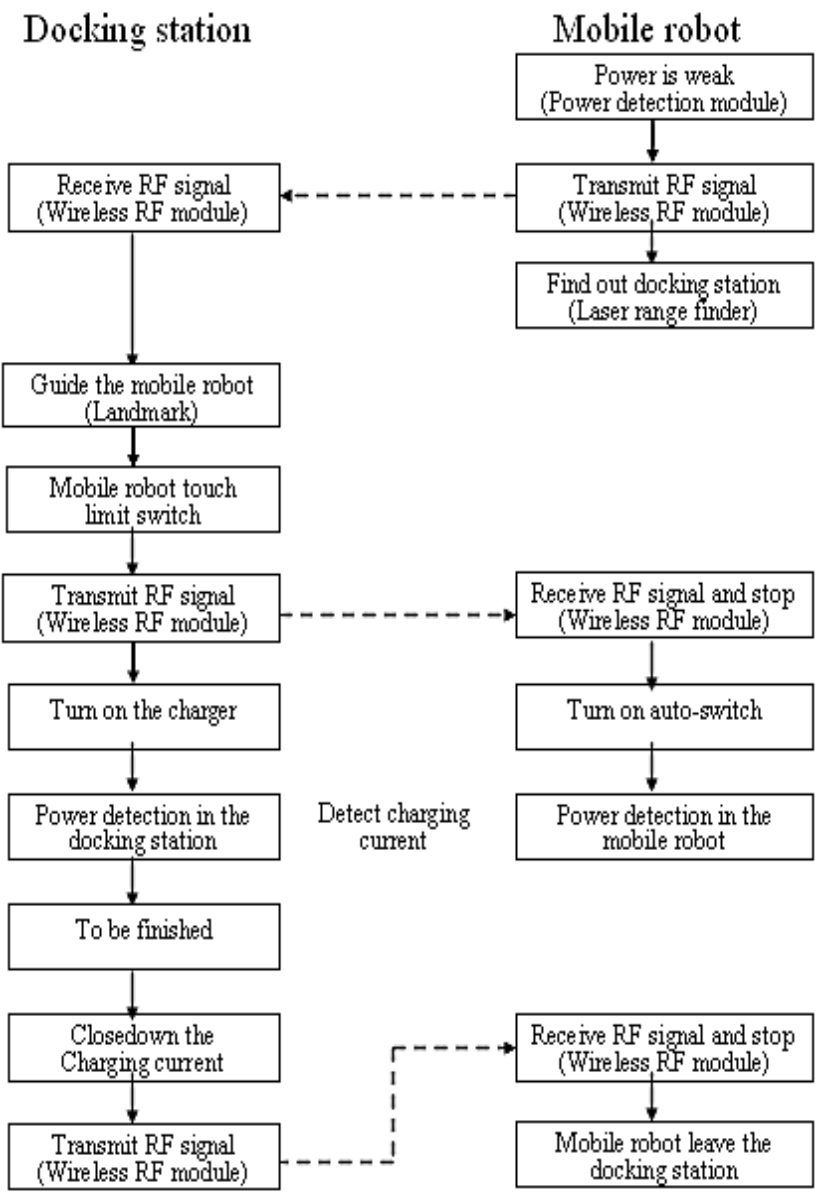

Figure 8. Auto-recharging Processing.

The mobile robot moves forward to the docking station and touches the limit switch. The controller of the docking station receives the signal from the limit switch and transmits the stopping command to the mobile robot via wireless RF interface. The mobile robot receives the wireless RF signal and stops on the front side of the docking station to open the auto-switch. The charger device of the docking station supplies the charging current to the mobile robot. In the charging process the power detection modules monitor power variations in the docking station and the mobile robot simultaneously [17]. Each power detection module detects the charging current when it is in an abnormal condition, and subsequently turns off the relay device in the docking station, or turns off the auto-switch in the mobile robot. Finally, the power of the mobile robot is perceived to be enough according to the signals of the two power 
detection modules. The docking station closes down the charging current from the charger, transmits an RF signal to the mobile robot, and triggers the mobile robot to leave the docking station. The mobile robot receives the command from the docking station and closes down the auto-switch to leave the docking station.

\section{Motion planning}

The motion planning system of the mobile robot is shown in Figure 9. We use an NI motion control card and Maxon drivers to control the two DC servomotors of the mobile robot. The mobile robot uses the laser range finder to search for the landmark of the docking station and programs the motion trajectory according to the location of the assigned docking station. In the landmark detection process of the docking station, the mobile robot moves about $2 \mathrm{~m}$ to the front side of the docking station, and the offset angle is about 2 degrees from the centre line of the docking station, as shown in Figure 10(a) and (b). The laser range finder measures the distance from the docking station to be about $2 \mathrm{~m}$, as shown in Figure 10(c). The peak position is the landmark. Then the mobile robot moves forward to the docking station and stops about $50 \mathrm{~cm}$ from the front side of the docking station. The experimental results are shown in Figure $10(d)$ and (e). The distance (peak position) between the mobile robot and the docking station is shown in Figure 10(f) and is detected by the laser range finder. The mobile robot adjusts the offset angle for docking to 1 degree of the centre line of the docking station. That is to say, the docking angle is between 89 degrees and 91 degrees. The mobile robot moves forward to the docking station successfully using the assigned docking angle, as shown in Figure 10(g) and (h). This manoeuvre is successful because the structure of the docking station has a 2 degree offset.

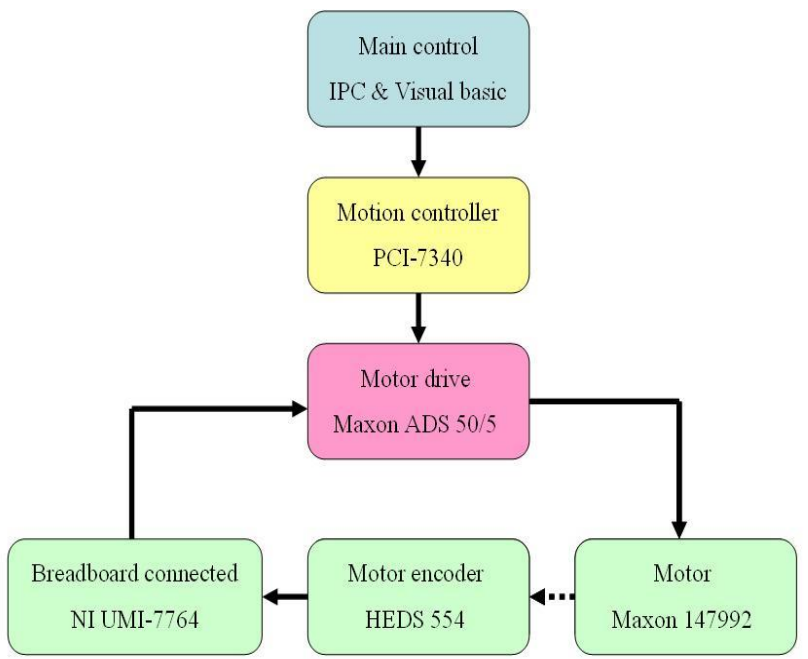

Figure 9. Motion Control Systems.

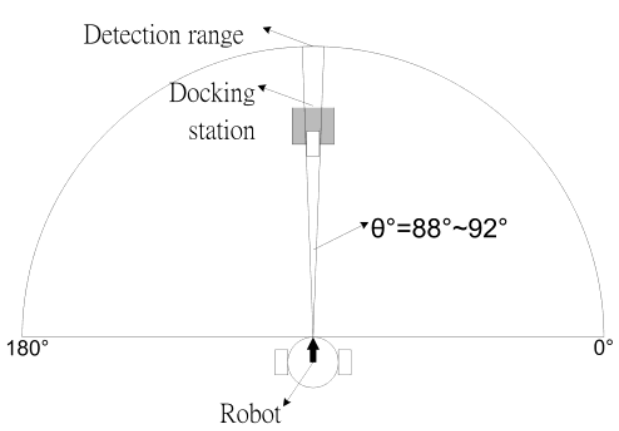

(a)

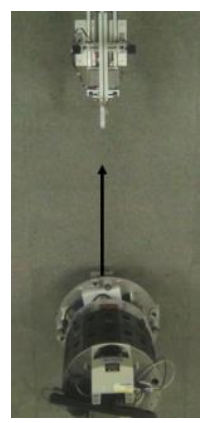

(b)

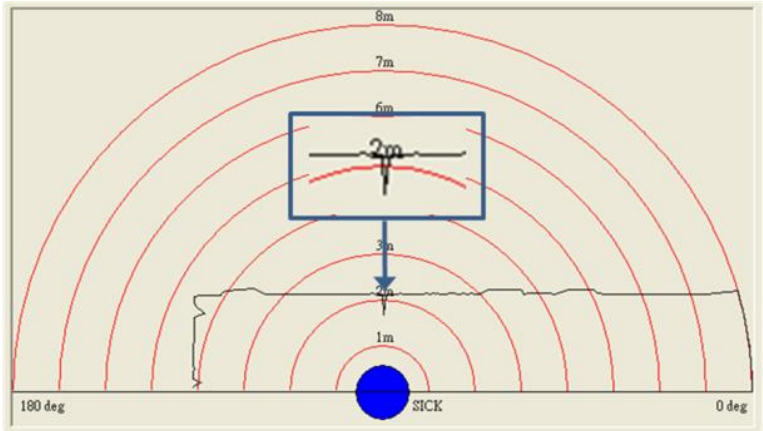

(c)

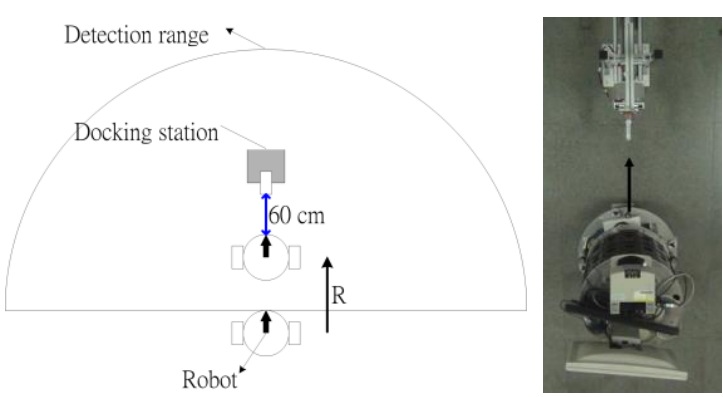

(d)

(e)

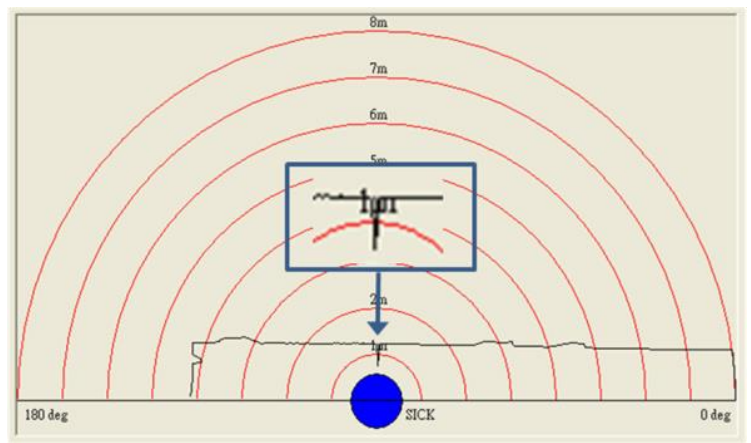

(f)

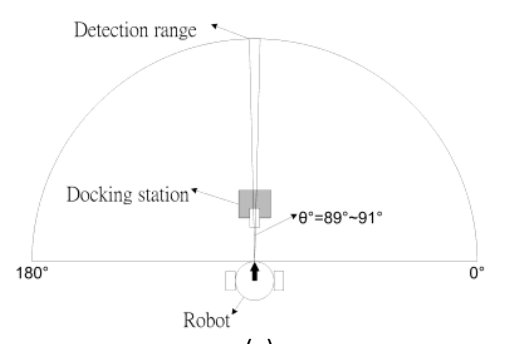

(g)

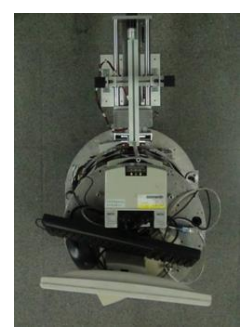

(h)
Figure 10. Motion Planning Method. 
We use two cases to explain the searching method for the mobile robot. In the first case, the mobile robot is located on the right side of the docking station, shown in Figure 11(a). In this instance the mobile robot uses the laser range finder to search for the landmark of the docking station. The user interface shows the landmark is in front of the mobile robot. The mobile robot calculates the displacement and orientation from the docking station and programs the motion trajectory. We can see the mobile robot turning left as $\theta$ and moving the displacement with $X \mathrm{~cm}$ in the horizontal direction. Then the mobile robot turns 90 degree left and moves forward to the docking station. Then the mobile robot moves to the front side of the docking station. The distance is about $2 \mathrm{~m}$. Finally, the mobile robot moves to touch the docking station using the previous process. The experiment scenario of the mobile robot presents the programmed motion trajectory shown in Figure 11(b).

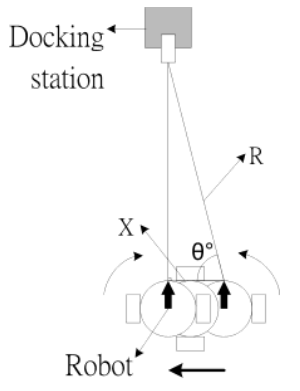

(a)

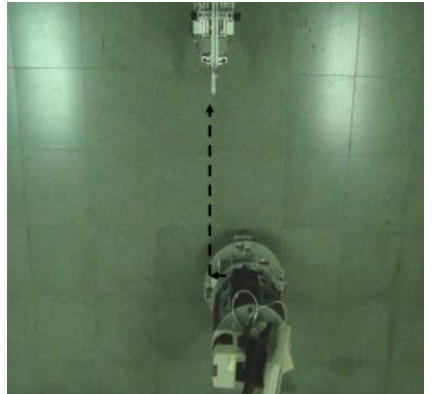

(b)
Figure 11. Auto-recharging Processing for Case I.

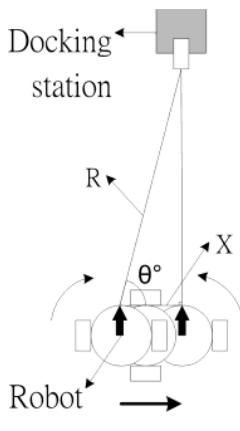

(a)

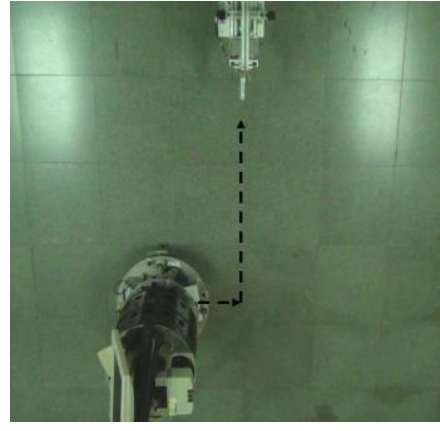

(b)
Figure 12. Auto-recharging Processing for Case II.

In the second instance, the mobile robot is located on the left side of the docking station, shown in Figure 12(a). The mobile robot uses the laser range finder to search for the landmark of the docking station. We can see that the distance between the mobile robot and the landmark of the docking station is detected at its minimum (peak position) using a laser range finder. The mobile robot calculates its displacement and orientation from the docking station. We can see that the mobile robot turns right with $\theta$ degrees and moves the displacement with $X \mathrm{~cm}$ in the horizontal direction.
Then the mobile robot turns 90 degree left and moves forward to the docking station. The distance $R$ is computed by the laser range finder of the mobile robot. Finally, the motion scenario of the mobile robot uses the programmed motion trajectory, shown in Figure 12(b).

\section{Algorithm analysis}

In power detection and fault isolation, we use a redundant management method and a statistical signal estimation method, to get an exact measured value, and to isolate faulty measurement values of current and voltage signals $[4,18]$. This redundant management method is defined as:

$$
\begin{gathered}
\hat{x}(k)=\frac{\sum_{i=1}^{l} m_{i}(k) I_{i}(k)}{\sum_{i=1}^{l} I_{i}(k)}, \\
I_{i}(k)=\sum_{j=1, j \neq i}^{l} f\left[\left|m_{i}(k)-m_{j}(k)\right| \leq\left(b_{i}(k)+b_{j}(k)\right)\right], i=1,2, \ldots, l, \\
f[*]=\left\{\begin{array}{l}
1, \text { if } * \text { is true } \\
0, \text { if } * \text { is false }
\end{array}\right.
\end{gathered}
$$

The estimated value $\hat{x}(k)$ is calculated using equation (1). The $m_{i}$ is the $i_{\text {th }}$ measurement value obtained through the sensors of the power detection module. $e_{i}$ is the $i_{\text {th }}$ measurement error, $b_{i}$ is the specified error bound of the measurement $m_{i}$ and $I$ is the numbers of measurement signals of the power detection module. The maximum value of $l_{i}$ is $I-1$ which is more accurate; otherwise the minimum value of $I_{i}$ is zero which serves as the error measurement value, and isolates the measurement value in order to calculate the estimated value on the sample time $k$.

Then we can calculate the residual working time using auto-regression methods and use the power values that are calculated by the power detection module of the mobile robot to estimate current and voltage values. We fit a second-order polynomial regression

$$
y=a_{0}+a_{1} x+a_{2} x^{2}+e .
$$

$a_{0}, a_{1}$ and $a_{2}$ are the coefficient values of the second-order equation. The sum of the squares of the error is

$$
S_{r}=\sum_{i=1}^{n}\left(y_{i}-a_{0}-a_{1} x_{i}-a_{2} x_{i}^{2}\right)^{2}
$$


To generate the least squares fit, we take the derivative of Equation (5) with respect to each of the unknown coefficients of the polynomial, and we can get the equation (6).

$$
\begin{aligned}
& \left.n a_{0}+\left(\sum x_{i}\right) a_{1}+\left(\sum x_{i}^{2}\right) a_{1}=\sum y_{i}\right] \\
& \left(\sum x_{i}\right) a_{0}+\left(\sum x_{i}^{2}\right) a_{1}+\left(\sum x_{i}^{3}\right) a_{2}=\sum x_{i} y_{i} \\
& \left(\sum x_{i}^{2}\right) a_{0}+\left(\sum x_{i}^{3}\right) a_{1}+\left(\sum x_{i}^{4}\right) a_{2}=\sum x_{i}^{2} y_{i}
\end{aligned}
$$

Finally we can calculate the coefficient values $a_{0}$, $a_{1}$ and $a_{2}$ from Equation (6). Then we set a power critical value to be $P_{s}$ to compute the residual working time of the mobile robot as follows :

$$
a_{2} x^{2}+a_{1} x+a_{0}=P_{s} .
$$

We can calculate the $x$ value (the unit is second) from Equation (7). The sample time of the main controller from the power detection module is 1 second. The residual working time is calculated using equation (8) as follows:

$$
x=\frac{-a_{1} \pm \sqrt{a_{1}^{2}-4 a_{2}\left(a_{0}-P_{s}\right)}}{2 a_{2}} .
$$

The $x$ value is under the threshold working time that is selected by the user. The mobile robot must execute the auto-recharging process. Otherwise the mobile robot will be shutdown. In the other case, the $x$ value does not exist. That is to say, the power is in an adequate condition. The mobile robot can't execute auto- recharge processing. The computation process is implemented in the main controller of the mobile robot. The main controller can receive the current and voltage measurement values from the power detection module via a series interface.

\section{User interface}

There are three user interfaces being developed in the mobile robot. These are the motion control interface, the auto-recharging monitoring system and the communication interface of the detection system. The user interface of the motion control system is classified in six parts for the mobile robot, as shown in Figure 13. The communication port between the user interface and mobile robots is 1 , and the baud-rate is set as 9600 . Part " $A$ " can set the scanning model of the laser range finder. The scanning mode can be adjusted for one scan, continuous scanning or stop scanning. Users can set the scanning object according to various motion modes of the mobile robot. The detection signals of the IR module and the compass module are displayed in part " $B$ ". IR sensors show that they have detected an obstacle by displaying a red symbol, and the compass sensor displays the orientation of the mobile robot.

Part " $\mathrm{C}$ " can control the mobile robot so that it moves forward or backward, turns right or left, and stops. The orientation and position of the docking station is detected by the laser range finder shown in part " $D$ ". Part "E" displays the outlook of the detected obstacle. The user interface sets the mobile robot to be the center of the region and displays the detected angle range from 0 degree to 180 degree, and the maximum distance range is $8 \mathrm{~m}$. The distance information of the detected obstacle is listed in part " $F$ " and is classified into four regions. Each region only displays the distance values of a 45 degree measurement range.

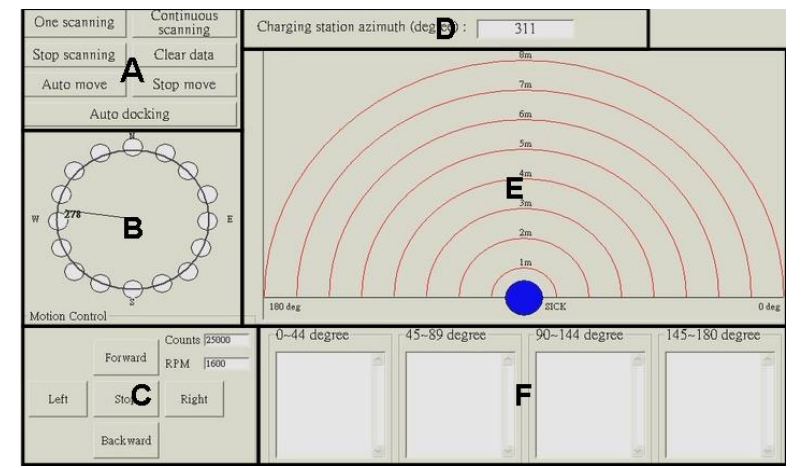

Figure 13. User Interface of the Motion Control System.

The user interface of the auto-recharging monitoring system combines the information of the docking station and the mobile robot and contains five parts, shown in Figure 14. Label " $A$ " displays the measurement values of current and voltage and computes the estimated value and average value of the power system using the proposed algorithms in the mobile robot. Label " $B$ " displays the charging process of the auto-recharging system and contains an alarm, indicates when charging starts and finishes, and displays the charging time and the total power output in the docking station. Label " $\mathrm{C}$ " displays real-time information during the charging process. Users can stop or restart the charging process, shown in label " $D$ ". Label " $E$ " plots the current curve and the voltage curve of the charging process in real-time in the docking station.

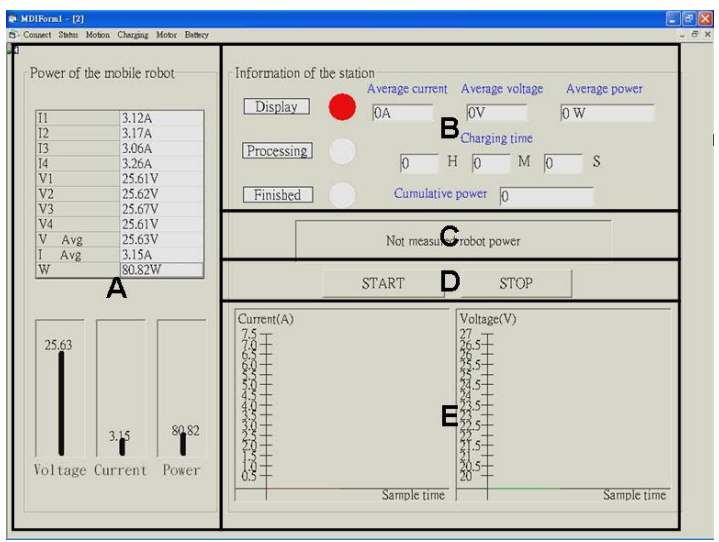

Figure 14. User Interface of the Auto-recharging Monitoring System. 
The communication interface of the detection system is shown in Figure 15 . The connection status of each detection module is displayed in part " $A$ ". It can display the information of the connected detection module. The information contains start bytes, ID codes, data numbers and check bytes. Users remove each connected detection module that is displayed in the user interface. The interface doesn't display the information of the removed module immediately. Part "B" displays the numbers of the total connected detection modules.

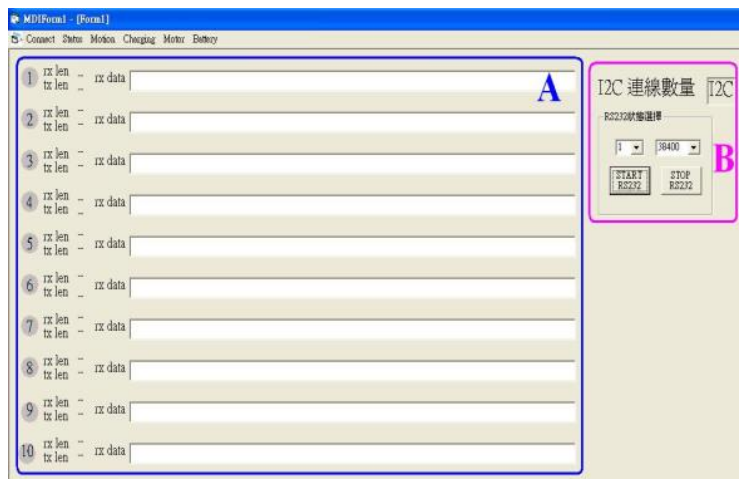

Figure 15. Communication Interface of the Detection System.

\section{Experimental results}

Under normal conditions the mobile robot moves autonomously in free space. The power detection module measures the power of the mobile robot at any time. If the power status is enough, the user interface displays a green symbol on its "Display", and the charging current and charging voltage is zero. The experimental result is shown in Figure 16. The mobile robot doesn't start the auto-recharging process, uses the proposed methods to detect variations in power, and predicts the residual working time continuously. We can see the outlook design of the mobile robot shown in the left side of Figure 16, and the power status of the mobile robot and the docking station is shown in the right side of Figure 16. The power of the docking station is zero, and no current is supplied to the mobile robot. In the residual power prediction experiment, the user can set the critical power. The main controller of the mobile robot calculates the residual working time using an autoregression method. Then it computes the residual working time to be under the threshold time. The mobile robot then transmits a charging command to the docking station and uses the laser range finder to search for the landmark of the docking station.

The mobile robot moves to the docking station and touches the connect pins and the limit switch. The power detection module of the docking station receives the signal of the limit switch, transmits an RF signal to the mobile robot, and provides the charging current to the mobile robot. The mobile robot receives the charging command from the docking station, stops on the front side of the docking station and prepares to execute the charging process. Then the power detection module turns on the auto-switch to receive the charging current from the charger of the docking station, and measures the charging current using the proposed methods. We can see the charging current of the charger being about $2.2 \mathrm{~A}$ and the voltage is $26 \mathrm{~V}$. The experimental result is shown in Figure 17.

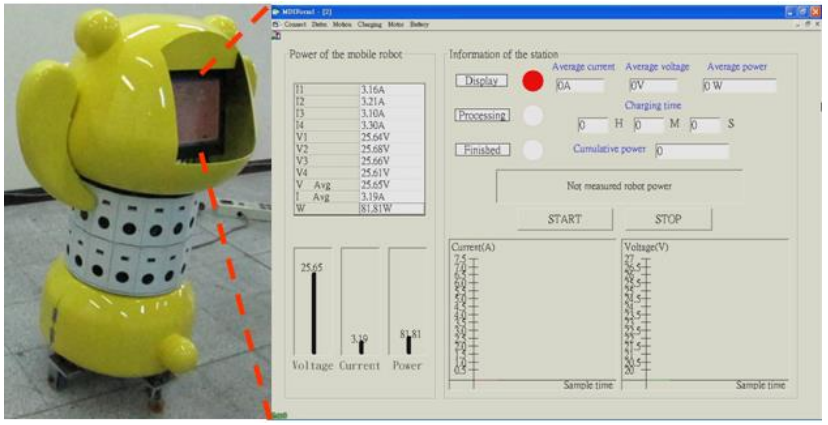

Figure 16. Starting to Charging Processing.

Then the power detection module measures the total current of the mobile robot to be about 3.15A and the voltage is determined to be about $25 \mathrm{~V}$. The total current includes the charging current and working current of the mobile robot. The main controller and some devices of the mobile robot must work in the charging process. The user interface of the mobile robot displays a green label on "Processing", shown in Figure 17. To determine the performance of the docking and charging process, 100 experimental results were performed. These results show a 99\% successful rate for the docking process. We may use the remote controller to stop, or restart the charging processing at any time to present abnormal conditions. The experimental results are almost always successful in the charging process. We can see the charging current being about $2.1 \mathrm{~A}$, which is displayed in the LCD panel of the power detection module, shown in the right side of Figure 17 . We can see the charging current being smaller than the original charging current for a long time. The charging current is detected by the two power detection modules to be smaller than the threshold value. The user interface displays that the charging processing is finished. The experimental results are shown in Figure 18. The docking station transmits a wireless RF signal to the mobile robot and closes the charging current. We can see the charging current being zero in the user interface and LCD panel of the power detection module. The mobile robot receives the wireless RF signal and closes the auto-switch to cut out the charging current. Then the mobile robot leaves the docking station shown in the left side of Figure 18. 


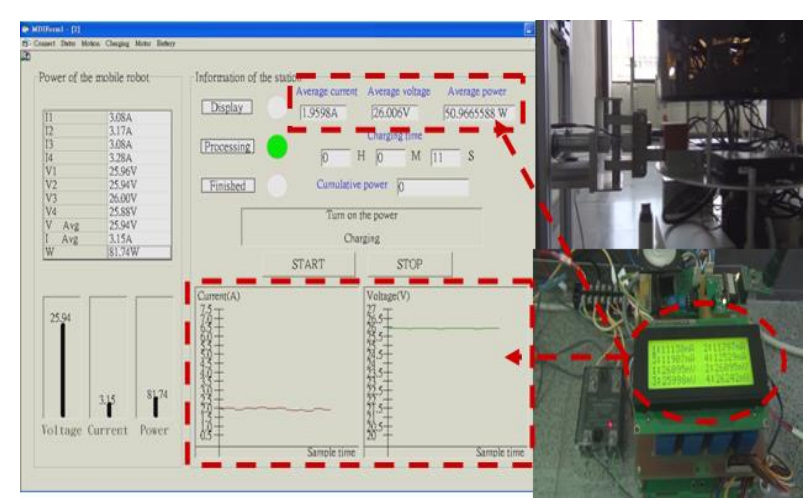

Figure 17. Charging Processing.

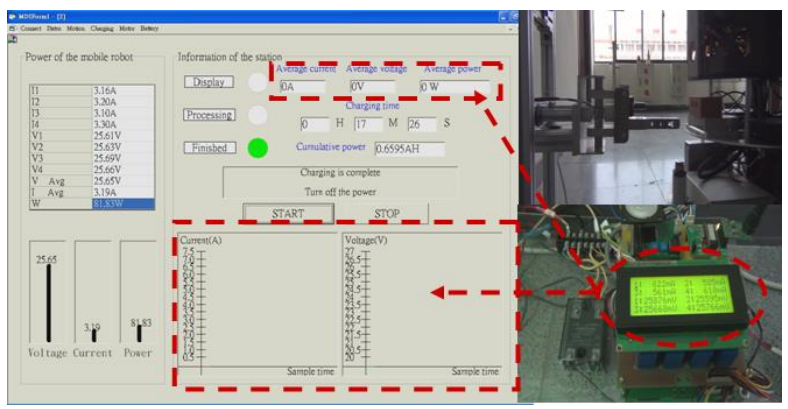

Figure 18. Charging Processing is Finished.

\section{Conclusion}

We have developed an auto-recharging monitoring system that has been integrated with a mobile robot and we have completed a series of auto-recharging processes. First, we designed a power detection module to measure current and voltage values and to compute variations in the power available to the mobile robot. Then the main controller of the mobile robot uses the power curve to calculate real-time residual power and working time. The detection, isolation and prediction algorithms use a redundant management method, a statistical signal detection method and an auto-regressive method. When the power of the mobile robot is under the threshold value for working a long time, the mobile robot uses a laser range finder to search for a docking station and moves to the docking station in response to a landmark. We designed a multiple DOFs docking station with an optimal docking angle for the mobile robot and programmed a new auto-recharging process to enhance the successful rate of the docking behavior. In the docking process, the mobile robot communicates with the docking station at any time via a wireless RF interface and knows the charging status during the recharging process. The auto-recharging monitoring system detects the charging current of the docking station and the mobile robot simultaneously and determines if the charging status is normal. That is to say, the mobile robot monitors the auto-recharging process and will deal with any dangerous conditions in real-time. Finally, the charger of the docking station provides the mobile robot with enough power. The mobile robot receives a command via a wireless RF interface and leaves the docking station. In the future, we will develop a remote monitoring system which can be integrated with the auto-recharging system.

\section{Acknowledgement}

This work "Development of An Intelligent Auto-recharging System," was supported by a grant from the National Science Council of Taiwan (NSC 101-2221-E-224-007).

\section{References}

[1] R. C. Luo, K. L. Su, and C. W. Deng, "Multisensor based power diagnosis system for an intelligent robot," in The 29th Annual Conference of the IEEE Industrial Electronics Society (IECON), Roanoke, Virginia, USA, 2003, vol. 3, pp. 2500-2505. doi: $10.1109 /$ IECON.2003.1280638

[2] K. L. Su, "Automatic fire detection system using adaptive fusion algorithm for fire fighting robot," in IEEE International Conference on Systems, Man and Cybernetics (SMC), Taipei, Taiwan, 2006, vol. 2, pp. 966-971. doi: $10.1109 /$ ICSMC.2006.384525

[3] T. L. Chien, H. Guo, K. L. Su, and S. V. Shiau, "Develop a multiple interface based fire fighting robot," in the 4th IEEE International Conference on Mechatronics (ICM), Kumamoto, Japan, 2007, pp. 1-6, WA1-B-3.

doi: 10.1109/ICMECH.2007.4280040

[4] K. L. Su, J. H. Tzou, and C. C. Liu, "Development of a multisensor-based residual power prediction system for mobile robots," in IEEE Workshop on Advanced Robotics and Its Social Impacts (ARSO), Hsinchu, Taiwan, 2007, pp. 114-119. doi: $10.1109 /$ ARSO.2007.4531424

[5] L. K. Horning, J. M. Soden, R. R. Fritzemeier, and C. F. Hawkins, "Measurements of quiescent power supply current for cmos ics in production testing," in International Test Conference, 1987, pp. 300-309.

[6] J. M. Soden and C. F. Hawkins, "Test considerations for gate oxide shorts in cmos ics," IEEE Design \& Test of Computers, vol. 3, no. 4, pp. 56-64, 1986. doi: $\underline{10.1109 / m d t .1986 .294977}$

[7] P. J. Liu, Y. H. Zhu, X. D. Cui, X. L. Wang, J. B. Yan and J. Zhao, "Multisensor-based autonomous docking for ubot modular reconfigurable robot," in International Conference on Mechatronics and Automation (ICMA), Chengdu, China, 2012, pp. 772-776.

doi: 10.1109/ICMA.2012.6283240 
[8] Y. N. Ren, W. Zou, H. Fan, A. X. Ye, K. Yuan and Y. $\mathrm{Ma}$, "A docking control method in narrow space for intelligent wheelchair," in International Conference on Mechatronics and Automation, Chengdu, China, 2012, pp. 1615-1620.

doi: $10.1109 /$ ICMA.2012.6284378

[9] H. Shen, X. Wang, J. Wang, Y. Xie, and W. Wang, "Vision based battery exchange robots for electric vehicle," in International Conference on Mechatronics and Automation (ICMA), Chengdu, China, 2012, pp. 2472-2476. doi: 10.1109/ICMA.2012.6285734

[10] Y. W. Bai and M. F. Hsueh, "Using an adaptive iterative learning algorithm for planning of the path of an autonomous robotic vacuum cleaner," in IEEE 1st Global Conference on Consumer Electronics (GCCE), Tokyo, Janpan, 2012, pp. 401-405.

doi: $10.1109 /$ GCCE.2012.6379640

[11] M. C. Silverman, D. Nies, J. Boyoon, and G. Sukhatme, "Staying alive: A docking station for autonomous robot recharging," in IEEE International Conference on Robotics and Automation (ICRA), Washington, DC, USA, 2002, vol. 1, pp. 1050-1055. doi: 10.1109/ROBOT.2002.1013494

[12] W. G. Walter, The living brain. New York: W. W. Norton, 1963.

[13] K. Roufas, Y. Zhang, D. Duff, and M. Yim, "Six degree of freedom sensing for docking using IR LED emitters and receivers," in The 7th International Symposium On Experimental Robotics (ISER), Hawaii, USA, 2000.

Available:

http://www.ri.cmu.edu/events/iser00/papers/rouf as.pdf
[14] R. C. Arkin and R. R. Murphy, "Autonomous navigation in a manufacturing environment," IEEE Transactions on Robotics and Automation, vol. 6, no. 4, pp. 445-454, 1990. doi: $10.1109 / 70.59355$

[15] B. W. Minten, R. R. Murphy, J. Hyams, and M. Micire, "Low-order-complexity vision-based docking," IEEE Transactions on Robotics and Automation, vol. 17, no. 6, pp. 922-930, 2001. doi: $10.1109 / 70.976026$

[16] N. Barnes and Z. Q. Liu, "Fuzzy control for active perceptual docking," in The 10th IEEE International Conference on Fuzzy Systems, Melbourne, Australia, 2001, vol. 3, pp. 1531-1534. doi: 10.1109/FUZZ.2001.1008954

[17] K. L. Su, S. H. Chia, J. H. Guo, and B. Y. Li, "Develop a power prediction system for scheduling loading of mobile robots," The Innovative Computing, Information and Control - Express Letters (ICIC-EL), vol. 5, no. 8(B), pp. 2791-2798, 2011.

[18] K. L. Su, J. H. Guo, C. W. Hung, and Y. C. Song, "Design an auto-recharging system for mobile robots," Applied Mechanics and Materials, vol. 190-191, pp. 666-672, 2012. doi: 10.4028/www.scientific.net/AMM.190-191.666 